\title{
Socio-Economic Differences in Early Initiation of Breastfeeding Among Children in a Ugandan Cross-Sectional Study
}

\author{
Ratib Mawa ${ }^{1, ~ *, ~ C a r o l i n e ~ K a m b u g u ~ N a b a s i r y e ~}{ }^{2}$, Margaret Chota ${ }^{1}$, Stephen Lawoko ${ }^{1}$, \\ Tracy Leigh Schumacher ${ }^{3}$, Krishna Nand Sharma ${ }^{1}$ \\ ${ }^{1}$ Department of Public Health, Faculty of Health Sciences, Victoria University Kampala, Uganda \\ ${ }^{2}$ Department of Nursing and Midwifery, Faculty of Health Sciences, Lira University, Lira, Uganda \\ ${ }^{3}$ Department of Rural Health, Faculty of Health and Medicine University of Newcastle, Newcastle, Australia
}

Email address:

ratib@vu.ac.ug (R. Mawa), leakycarolyn326@gmail.com (C. K. Nabasirye), chotamargret@yahoo.com (M. Chota), deanhealthscience@vu.ac.ug (S. Lawoko), tracy.schumacher@newcastle.edu.au(T. L. Schumacher), vicechancellor@vu.ac.ug (K. N. Sharma)

\section{To cite this article:}

Ratib Mawa, Caroline Kambugu Nabasirye, Margaret Chota, Stephen Lawoko, Tracy Leigh Schumacher, Krishna Nand Sharma. SocioEconomic Differences in Early Initiation of Breastfeeding Among Children in a Ugandan Cross-Sectional Study. Journal of Food and Nutrition Sciences. Vol. 7, No. 1, 2019, pp. 8-15. doi: 10.11648/j.jfns.20190701.12

Received: March 28, 2019; Accepted: May 5, 2019; Published: May 23, 2019

\begin{abstract}
Early initiation of breastfeeding reduces neonatal and maternal morbidity and mortality, enhances mother-infant dyad bonding and maternal optimal breastfeeding practices. Studies on socio-economic inequalities in early initiation of breastfeeding are sparse in Uganda. This study therefore aimed to examine the association between socio-economic status and early initiation of breastfeeding among 5504 children aged 0-23 months in a country-wide population based cross-sectional study. Early initiation of breastfeeding defined as initiation of breastfeeding within an hour of birth was the outcome of interest. Proxy measures of socio-economic status (maternal occupation and household wealth index) were the exposure variable of interest. Bivariate analysis was conducted to determine the distribution of the prevalence of early initiation of breastfeeding by infant-mother dyad characteristics. Multivariate binary logistic regression models adjusted for relevant confounders were fitted to examine the association between socio-economic status and early initiation of breastfeeding. The results showed an overall prevalence early initiation of breastfeeding of $67.8 \%$ among Ugandan children. Children whose mothers self-reported their occupation as farmers and skilled manual workers had lower odds of early initiation of breastfeeding compared to those whose mothers were doing professional/technical/managerial work; OR: $0.69(0.51-0.95)$ and $0.70(0.49-0.99)$ respectively. On the other hand, children that lived in the poorest and poorer households had lower odds of early initiation of breastfeeding OR: $0.46(0.32-0.67)$ and $0.64(0.43-0.94)$ respectively. In conclusion, we found individual and household socio-economic inequality in EIB among children less than two years of age. Targeting interventions for promotion of EIB to less affluent mother-child dyads and households might be important in increasing optimal breastfeeding practices.
\end{abstract}

Keywords: Children, Breastfeeding, Socio-Economic Status, Uganda, Africa

\section{Introduction}

The benefits of early initiation of breastfeeding (EIB) to neonatal health and survival are well established [1]. EIB is known to reduce all cause and infectious disease specific morbidity and mortality mainly attributed to the protective effect of the nutrient dense and antibody rich first maternal breast milk (colostrum) [2-7]. EIB is also known to reduce postpartum hemorrhage, stimulate breast milk production, promote mother-neonate bonding and enhances optimal breastfeeding practices $[5,8,9]$. The aversion of approximately $22-44 \%$ neonatal deaths and the significant reduction in maternal morbidity and mortality attributed to EIB [10-14] is important for child survival especially in 
developing countries with limited capacity to implement a wide-range of child survival interventions. Despite of these known benefits of EIB, across the globe less than half (42\%) of the newborns are breastfed within an hour of birth [15, 16]. In sub-Saharan Africa, a cross-sectional study based on nationally representative demographic and health surveys in 29 countries, conducted between 2010 and 2015 found an overall prevalence of EIB of $37.84 \%$ [17], lower than the global average. On the contrary the prevalence of EIB among children under-five years of age in Uganda is approximately twice higher than that of the global and sub-Saharan African averages, however a large proportion of newborns (33\%) [18], are not breastfed within an hour of birth. To enhance reduction of all cause and infectious disease specific neonatal and infant morbidity and mortality in Uganda, there is need for EIB for all newborns as recommended by the World Health Organization (WHO) [19]. Achieving this goal requires identification of factors inhibiting EIB for newborns to facilitate development of effective preventive interventions.

Socio-economic inequality in EIB creates differential distribution of the benefits of EIB among mother-infant dyads, contributing to inequality in neonatal and infant morbidity, mortality. Observational studies conducted in subSaharan Africa have shown the existence of socio-economic inequalities in EIB. A cross-sectional study conducted in Ethiopia found a positive association between maternal education, unemployment and EIB respectively and no association between household wealth status and EIB among children less than 24 months of age [20]. On the same note, another cross-sectional study conducted in a semi-urban area in Nigeria, found lower likelihood of EIB among mothers with no formal or primary level education compared to those that had attained tertiary level education [21]. Likewise a Nepalese study based on analysis of data from three demographic and health surveys found that children whose mothers had primary, secondary and tertiary level education had higher odds of EIB compared to those whose mothers had no formal education [22]. These findings demonstrate social patterning in EIB for newborns in developing countries. The pathways through which household or maternal socio-economic status influence EIB are not clearly delineated, however it might follow earlier-on described models on social determinants of health inequalities and health [23, 24].

er than socio-economic status at individual or household level, other factors that were found to influence EIB in developing and developed countries can be grouped into socio-demographic, medical, psycho-social, cultural and policy related factors [25-29]. Of interest to mention among others are those factors that are positively associated with EIB, which include but not limited to maternal-newborn skin-to-skin contact after birth, health facility based deliveries that are supervised by skilled attendants, maternal age above 30 years and normal vaginal delivery. Factors that are negatively associated with EIB include maternal multiparity and living in rural areas [20].
Epidemiological studies on socio-economic inequalities in EIB are scarce in Uganda. The few studies known to us had either methodological issues or were conducted in institutional or localized geographical area settings, making either the validity of the results questionable or limiting generalization of results to all mother-child dyads nationwide. A cross-sectional study based on secondary data from the Uganda demographic and health survey reported a positive association between maternal education and EIB, however it's unclear what comparator and effect measure was used and reported respectively making the validity of the results questionable [30]. Another cross-sectional study that was conducted among mother-infant dyads at Mulago National referral hospital did not either adjust for or include measures of socio-economic status in their analysis but rather identified maternal ante-natal care attendance, HIV positive sero-status, negative cultural believes and caesarean section deliveries as the factors negatively associated with EIB [31]. Hence it's unclear whether socioeconomic inequality in EIB exists among newborns in Uganda. Identifying social differences in EIB is therefore essential for targeting interventions for promotion of optimal breastfeeding practices and policy modification that will eventually enhance prospects for achieving health equity among Ugandan mothers and children. This study therefore examined socio-economic inequality in EIB among children less than two years in the general population in Uganda.

\section{Methods and Materials}

\subsection{Setting and Materials}

The materials for this study were derived from Uganda, a land locked developing country located in East Africa with a projected population of approximately 41 million people. Uganda's has a weak healthcare system with huge gaps in human resource, drugs/medical equipment's, quality of services and financing. Healthcare and nutrition specific services are delivered through static health facilities that ranged from health center II (lowest level) to the national referral hospital located in Kampala, the capital city. For this study, secondary data from the 2016 Uganda Demographic and Health Survey (UDHS) was used. The UDHS is a USAID sponsored five year interval national survey that started since 1988/89. It's used to obtain information on health and social development indicators of national importance. The UDHS employs a two-stage cluster sampling design to select sample households from which eligible women and men were interviewed. In 2016 UDHS sampling process, first the 2014 census enumeration areas for Uganda were selected, followed by selection of a random sample of households in the second stage. Participants included Women aged 15- 49 years and a sub-sample of men aged 18-54 years were eligible. The survey response rate for women and men was very high i.e. $97 \%$ and $94 \%$ respectively [32]. 


\subsection{Study Design and Population}

This is a cross-sectional study based on secondary data. A total population of 5504 (weighted $=5417$ ) children aged 0-23 months were selected from the dataset and analyzed. Only the youngest child for each interviewed mother, who were alive at time of the survey and had complete data on timing of initiation of breastfeeding after birth were included. For details on the inclusion and exclusion criteria applied, please refer to figure 1. This group of children was chosen based on the WHO guidelines on infant and young child feeding practices [33] and the Ugandan Government ministry of health's policy guidelines on infant and young child feeding practices [34]. For details on the inclusion and exclusion criteria applied, refer to the figure 1 below.

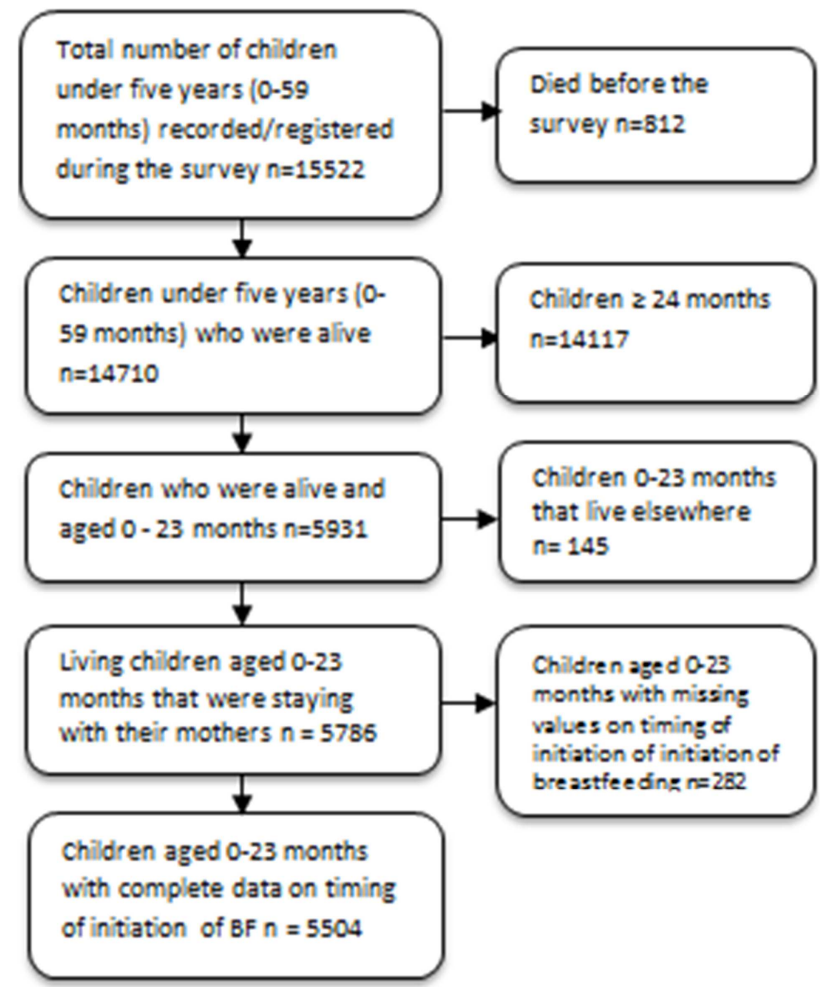

Figure 1. Schematic selection process of the study population.

\subsection{Exposure and Outcome}

\subsubsection{Socio-Economic Status}

Two proxy measures of socio-economic status were used; maternal occupation and household wealth index. Data on measures of socio-economic status was collected using the validated 2016 UDHS women's questionnaire. By answering the question; what is your occupation? i.e. what kind of work do you mainly do? Mothers stated the type of work they do to earn living. Maternal occupation defined and recorded based on the international classification of occupations [35]. For the purpose of having adequate number of subjects in each occupational category, the variable maternal occupational was re-grouped into seven categories; 1) professionals/technical/managerial, 2) Not in the labor force (not working), 3) clerical/sales, 4) agriculture (self-employed
\& employee), 5) household/domestic/services providers, 6) skilled manual workers and 7) unskilled manual workers. Professionals/technical/managerial occupation category was used as the reference category in the analysis. On the other hand household wealth index, a measure of a household's cumulative living standard, was calculated based on household asset ownership. Information was collected on household ownership of assets such as cars and televisions, house characteristics (such as type of wall, floor materials etc.), drinking water sources, toilet facilities, etc. Each household item was then assigned a factor score generated through principle component analysis [36, 37]. Individuals were then ranked according to summed-up household scores after which quintile was created ranking individuals into $20 \%$ poorest households to $20 \%$ richest households.

\subsubsection{Early Initiation of Breastfeeding}

Early initiation of breastfeeding (EIB) was defined as putting the newborn to breastfeed within an hour of birth [38]. Self-reported EIB for their newborn after birth was assessed through the standardized 2016 UDHS women's questionnaire. By answering the question; how long after birth did you put (NAME) to breastfeed? Mothers stated the time for initiation of breastfeeding for their children after birth. Their responses were recorded as; 1) put to breastfeed immediately after birth, 2) within an hour, 3) in hours, 4) on the first day and 5) in more than a day. In the analysis, we then created a binary variable "early initiation of breastfeeding"; recorded as 1 if the child was breastfed within 1 hour of birth and 0 otherwise [39].

\subsection{Potential Confounders}

The following variables were included as potential confounding variables based on published literature on their association with EIB. Childs sex (recorded as 1 if male \& 0 otherwise), child's birth weight ( 1 if the child had $\geq 2500$ grams and 0 otherwise), child's age (recorded into 12 categories 2 year interval), place of delivery (three categories; home, health facility \& others), type of delivery ( 1 caesarean section delivery and 0 otherwise), maternal age (seven categories of 5 year intervals), maternal education (four categories; no education, primary, secondary \& tertiary level), maternal marital status (four categories; never married, married, living with a partner and widow/divorced/separated), paternal education (four categories; no education, primary, secondary and tertiary), type of place of residence (two categories; rural and urban), maternal health insurance (1 insured and 0 otherwise [40]. All the above potential confounding variables were measured using the standardized UDHS questionnaires.

\subsection{Statistical Analysis}

To account for the effect of clustering and stratification introduced by the survey design, complex survey data analysis procedures were followed. Bivariate analysis was conducted to calculate the prevalence of EIB and the distribution of study subjects by socio-demographic 
characteristics. To calculate the crude and adjusted odds ratios and their corresponding 95\% confidence for EIB, binary logistic regression models were fitted and adjusted for important confounders. Subjects with missing values for the outcome variable (EIB), and the exposure variables (household wealth index and maternal occupation) were excluded from the analysis. The statistical analysis was performed using SPSS version 24.

\subsection{Ethical Consideration}

Ethical approval of the 2016 UDHS was done by ICF International (Calverton, MD, USA) Institutional Review Board (IRB). During the 2016 UDHS conduct, women and men that participated in the survey provided informed written consent. The survey participants were assured of no harm in participation and neither was any harm to the study subjects reported in the final 2016 UDHS. For purpose of this study, the secondary data was obtained after written approval by DHS program archivist, authorization number 117079 .

\section{Results}

As shown on Table 1, 50.7\% of the children were boys; majority (94.1\%) had normal birth weight. The distribution of the study subjects by age was relatively uniform, however slightly higher proportion of children were in the age group 8-9 months and the lowest proportion was in the age group of 22-23 years of age. Most $(76.3 \%)$ of the children were delivered in health facilities, supervised by trained medical personnel. Majority of the mothers $(65.6 \%)$ attained primary level education at the time of the survey and most of them were either living with a partner or married. Most mothers reported their occupation as farmer. On the hand most children had fathers that attained primary level education at the time of the survey conduct in 2016. Majority of the children lived in the poorest households and in rural areas with no health insurance. The overall prevalence of EIB was $67.8 \%$. Children whose mothers reported not working (not in the labor force) had the highest prevalence of EIB and those whose mothers were employed in the agricultural sector had the lowest prevalence $(64.9 \%)$ of EIB. Similarly the highest prevalence $(73.1 \%)$ of EIB was among mother-child dyad that lived in the richest households and the lowest $(60.6 \%)$ was among those that lived in the poorest households. The crude and the adjusted odds ratios and their corresponding 95\% confidence interval for EIB by maternal occupation and household wealth index are shown on Table 2 and Table 3 respectively. Compared to children whose mothers had profession/technical/ managerial occupations, those whose mothers were not employed higher odds of EIB (OR; 1.10 CI (0.75 - 1.54). While those whose mothers were doing clerical/sales, agricultural, household/domestic/services, skilled manual and unskilled manual work had lower odds of EIB. At household level, children in the richer, middle, poorer and poorest households had lower odds of EIB compared to those in the richest households.

Table 1. Socio-demographic characteristics of 5417 (un-weighted 5504) children aged 0-23 months by early initiation of breastfeeding status.

\begin{tabular}{|c|c|c|c|}
\hline \multirow{2}{*}{ Mother-Infant dyad characteristics } & \multicolumn{3}{|c|}{ Initiation of breastfeeding (children aged 0-23 months) } \\
\hline & Total (\%) & Within 1 hour & Beyond 1 hour \\
\hline \multicolumn{4}{|l|}{ Child sex } \\
\hline Male & $2745(50.70)$ & $1870(68.10)$ & $0875(31.90)$ \\
\hline Female & $2672(49.30)$ & $1802(67.40)$ & $0870(32.60)$ \\
\hline Total & $5417(100)$ & $3672(67.80)$ & $1745(32.20)$ \\
\hline \multicolumn{4}{|l|}{ Birth weight (grams) } \\
\hline$<2500$ & $0318(05.90)$ & $0188(59.10)$ & $0130(40.90)$ \\
\hline$\geq 2500$ & $5097(94.10)$ & $3483(68.30)$ & $1614(31.70)$ \\
\hline \multicolumn{4}{|l|}{ Child`s age (Months) } \\
\hline $0-1$ & $427(7.90)$ & $289(67.70)$ & $138(32.30)$ \\
\hline $2-3$ & $485(9.00)$ & $340(70.10)$ & $145(29.90)$ \\
\hline $4-5$ & $446(8.20)$ & $324(72.60)$ & $122(27.40)$ \\
\hline 6-7 & $506(9.30)$ & $342(67.60)$ & $164(32.40)$ \\
\hline $8-9$ & $522(9.50)$ & $360(69.00)$ & $162(31.00)$ \\
\hline $10-11$ & $515(9.50)$ & $343(66.60)$ & $172(33.40)$ \\
\hline $12-13$ & $454(8.40)$ & $295(65.00)$ & $159(35.00)$ \\
\hline $14-15$ & $390(7.20)$ & $264(67.70)$ & $126(32.30)$ \\
\hline $16-17$ & $474(8.80)$ & $306(64.60)$ & $168(35.40)$ \\
\hline 18-19 & $440(8.10)$ & $305(69.30)$ & $135(30.70)$ \\
\hline $20-21$ & $386(7.10)$ & $261(67.60)$ & $125(32.40)$ \\
\hline $22-23$ & $371(6.90)$ & $242(65.20)$ & $129(34.80)$ \\
\hline \multicolumn{4}{|l|}{ Place of delivery } \\
\hline Home & $1193(22.00)$ & $0749(62.80)$ & $0444(37.20)$ \\
\hline Health facility & $4133(76.30)$ & $2876(69.60)$ & $1257(30.40)$ \\
\hline Others & $0090(01.70)$ & $0046(51.10)$ & $0044(48.90)$ \\
\hline CS Delivery & & & \\
\hline
\end{tabular}




\begin{tabular}{|c|c|c|c|}
\hline \multirow{2}{*}{ Mother-Infant dyad characteristics } & \multicolumn{3}{|c|}{ Initiation of breastfeeding (children aged 0-23 months) } \\
\hline & Total (\%) & Within 1 hour & Beyond 1 hour \\
\hline No & $5008(92.80)$ & $3510(70.10)$ & $1498(29.90)$ \\
\hline Yes & $0391(07.20)$ & $0151(38.60)$ & $0240(61.40)$ \\
\hline \multicolumn{4}{|l|}{ Maternal age (years) } \\
\hline $15-19$ & $0625(11.50)$ & $0384(61.40)$ & $241(38.60)$ \\
\hline $20-24$ & $1645(30.40)$ & $1088(66.10)$ & $557(33.90)$ \\
\hline $25-29$ & $1351(24.90)$ & $0965(71.40)$ & $386(28.60)$ \\
\hline $30-34$ & $0961(17.70)$ & $0652(67.80)$ & $309(32.20)$ \\
\hline $35-39$ & $0586(10.80)$ & $0415(70.80)$ & $171(29.20)$ \\
\hline $40-44$ & $0215(4.00)$ & $0148(68.80)$ & $067(31.20)$ \\
\hline $45-49$ & $0035(0.60)$ & $0020(57.10)$ & $015(42.90)$ \\
\hline \multicolumn{4}{|l|}{ Maternal Education Level } \\
\hline No education & $0525(09.70)$ & $0394(75.00)$ & $0131(25.00)$ \\
\hline Primary & $2154(65.60)$ & $1128(34.40)$ & $1128(34.40)$ \\
\hline Secondary & $1214(22.40)$ & $0858(70.70)$ & $0356(29.30)$ \\
\hline Tertiary & $0395(07.30)$ & $0265(67.10)$ & $0130(32.90)$ \\
\hline \multicolumn{4}{|l|}{ Maternal Current Marital Status } \\
\hline Never married & $0321(5.90)$ & $0203(63.20)$ & $118(36.80)$ \\
\hline Married & $2200(40.60)$ & $1463(66.50)$ & $737(33.50)$ \\
\hline Living with a partner & $2408(44.50)$ & $1664(69.10$ & $744(30.90)$ \\
\hline Widow/Divorced/Separated & $0487(9.00)$ & $0341(70.00)$ & $146(30.00)$ \\
\hline \multicolumn{4}{|l|}{ Maternal occupation } \\
\hline Not in labor force & $1028(19.00)$ & $0756(73.50)$ & $272(26.50)$ \\
\hline Professional/Technical/Managerial & $0380(07.00)$ & $0264(69.50)$ & $116(30.50)$ \\
\hline Clerical/Sales & $0514(09.50)$ & $0357(69.50)$ & $157(30.50)$ \\
\hline Agriculture (self-employed/employee) & $2428(44.90)$ & $1575(64.90)$ & $853(35.10)$ \\
\hline Household/domestic/services & $0276(5.10)$ & $0192(69.60)$ & $084(30.40)$ \\
\hline Skilled manual & $0614(11.30)$ & $0405(66.00)$ & $209(34.00)$ \\
\hline Unskilled manual & $0170(03.10)$ & $0120(70.60)$ & $050(29.40)$ \\
\hline \multicolumn{4}{|l|}{ Paternal Education level } \\
\hline No education & $301(6.70)$ & $0229(76.10)$ & $072(23.90)$ \\
\hline Primary & $2431(54.20)$ & $1598(65.70)$ & $833(34.30)$ \\
\hline Secondary & $1210(27.00)$ & $0845(69.80)$ & $365(30.20)$ \\
\hline Tertiary & $0547(12.20)$ & $0367(67.10)$ & $180(32.90)$ \\
\hline \multicolumn{4}{|l|}{ Household Wealth index } \\
\hline Poorest & $1230(22.70)$ & $745(60.60)$ & $485(39.40)$ \\
\hline Poorer & $1158(21.40)$ & $759(65.50)$ & $399(34.50)$ \\
\hline Middle & $1042(19.20)$ & $720(69.10)$ & $322(30.90)$ \\
\hline Richer & $0942(17.40)$ & $685(72.70)$ & $257(27.30)$ \\
\hline Richest & $1043(19.30)$ & $762(73.10)$ & $281(26.90)$ \\
\hline \multicolumn{4}{|l|}{ Type of place of residence } \\
\hline Urban & $1147(21.20)$ & $0838(73.10)$ & $0309(26.90)$ \\
\hline Rural & $4268(78.80)$ & $2833(66.40)$ & $1435(33.60)$ \\
\hline \multicolumn{4}{|l|}{ Insurance for respondent } \\
\hline Not insured & $5344(98.70)$ & $3627(67.90)$ & $1717(32.10)$ \\
\hline Insured & $0072(1.30)$ & $0044(61.10)$ & $0028(38.90)$ \\
\hline
\end{tabular}

Note: weighted totals might not add-up to the sample size across different characteristics due to missing values.

$\mathrm{NA}=$ Not available

Table 2. Crude and adjusted odds ratio (OR) and 95\% Confidence Interval (CI) for early initiation of breastfeeding for 5417 (Unweighted 5504$)$ children aged 0-23 months by maternal occupation.

\begin{tabular}{lll}
\hline \multirow{2}{*}{ Maternal Occupation } & Early Initiation of Breastfeeding & Adjusted OR (95\% CI) \\
\cline { 2 - 3 } & Crude OR (95\% CI) & 1.00 \\
\hline Professional/Technical/Managerial & 1.00 & $1.10(0.75-1.54)$ \\
Not in labor force & $1.22(0.86-1.72)$ & $0.95(0.64-1.41)$ \\
Clerical / Sales & $1.00(0.70-1.44)$ & $0.69(0.51-0.95)$ \\
Agricultural work & $0.81(0.61-1.08)$ & $0.89(0.56-1.44)$ \\
Household / Domestic / Services & $1.00(0.64-1.56)$ & $0.70(0.49-0.99)$ \\
Skilled Manual & $0.85(0.62-1.17)$ & $0.86(0.52-1.43)$ \\
Unskilled Manual & $1.05(0.65-1.70)$ & \\
\hline
\end{tabular}

OR Adjusted for caesarean section delivery, child sex, birth weight, child age, place of delivery, antenatal care attendance, health insurance 
Table 3. Crude and adjusted odds ratio (OR) and 95\% Confidence Intervals (CI) for early initiation of breastfeeding for 5417 (Unweighted 5504$)$ children aged 0-23 months by household wealth index.

\begin{tabular}{lll}
\hline \multirow{2}{*}{ Household Wealth Index } & \multicolumn{2}{l}{ Early Initiation of Breastfeeding } \\
\cline { 2 - 3 } & Crude OR $\mathbf{( 9 5 \%}$ CI) & Adjusted OR (95\% CI) \\
\hline Richest & 1.00 & 1.00 \\
Poorest & $0.57(0.44-0.73)$ & $0.46(0.32-0.67)$ \\
Poorer & $0.70(0.54-0.91)$ & $0.64(0.43-0.94)$ \\
Middle & $0.82(0.64-1.06)$ & $0.76(0.53-1.07)$ \\
Richer & $0.98(0.76-1.28)$ & $0.83(0.59-1.16)$ \\
\hline
\end{tabular}

OR adjusted for child sex, birth weight, age, mode of delivery, place of delivery, maternal age, maternal education level, maternal occupation, paternal education level, health insurance, and type of place of residence.

\section{Discussion}

The findings of this study revealed existence of socioeconomic inequality in EIB at both individual and household level. When maternal occupation was used as the proxy measure of socio-economic status, children whose mothers reported not working (not in the labor force) were $10 \%$ more likely to experience EIB compared to those whose mothers had Professional/Technical/Managerial professions; however this relationship was not statistically significant before and after adjusting for various confounding variables. This finding might imply the possibility of misclassification of EIB with respect to maternal occupation given that women who reported not being in the labor force are more likely members of different occupational groups' hence different socio-economic status. Similarly we also found no relationship between EIB and maternal occupation when children whose mothers worked as clerical/sales, household / domestic / services and unskilled manual were compared to those whose mothers had professional/technical and managerial occupations. These occupational groups tend to have low income in Ugandan context, therefore mother-child dyads are classified as having low socio-economic status. The reduced odds of EIB for children whose mothers belonged to these social groups might be linked to limitation in access to maternal healthcare services especially antenatal care and health facility based deliveries supervised by trained medical personnel. However the volatility of earnings received by women in these occupational groups in Uganda makes majority of mothers to engage in doing two or more jobs or occupations, hence misclassification of EIB by maternal occupation cannot be ruled out. Nevertheless we found an association between maternal occupation and EIB when children whose mother's identified their occupational group as farmers and skilled manual workers were compared to those whose mothers had professional/managerial and technical occupation. This might be because mothers in these occupational groups are low income earners and are similarly less likely have access to antenatal care services and health facility based delivery. The findings of this study are not in agreement with results of a Namibian cross-sectional study based on three waves of demographic and health survey data for the year 2000, 2006/2007 and 2013 [41]. In this study, authors found no association between maternal occupation and EIB when children whose mothers were farmers and those not working were compared to those whose mothers had paid jobs [41]. The disparity might be linked to residual confounding and misclassification since the Authors of the Namibian study lambed all paid occupational groups into one category. On the same note our results are also not consistent with those of a Nigerian cross-sectional study that found mixed results with occupational group of sales having association with EIB and agriculture (farmers) having no association with EIB [42]. The difference in the results of our study and the Nigerian one might lie in variation of potential confounding variables adjusted for and the maternal occupational categories used as the references in the analysis i.e. the Nigerian study used maternal occupational group of not working (not in the labor force) as their reference category in their analysis while in our study we used maternal occupational group of professional/technical/managerial as the reference category.

When household socio-economic differences in EIB among children was assessed, the findings showed that children whose mothers lived in the poorest, poorer, middle and richer households were 54, 36, 24 and $17 \%$ less likely to experience EIB compared to their counterparts in the richest households respectively. This inverse relationship between EIB and household socio-economic status showed statistical significant association only when children in the poorest and poorer households were compared to those in richest households with respect to EIB. This poor - rich differences in EIB among children might be linked to issues of access to maternal antenatal and delivery services [43], given that pregnant mothers in affluent families might be more likely to deliver in health facilities because they have financial capacity to access maternal healthcare services regardless of where they live in the country as opposed to their counterparts in poorer and poorest households that might have financial challenges limiting their access to antenatal care and health facility based deliveries that supervised by trained healthcare professionals. Our findings are consistent with the results of a Nigerian cross-sectional study which showed an increasing trend in EIB with increasing household socio-economic status [42], however they are not consistent with the results of an Ethiopian cross-sectional study that found no association between household socio-economic status and EIB [20]. This difference to a large extend reflect variations in confounding variables adjusted for in both 
studies and the references categories for the variable household wealth index employed for comparability of the effect measure for EIB among children.

\section{Conclusion}

We found individual and household socio-economic inequality in EIB among children less than two years of age in Uganda. Targeting interventions for promotion of EIB to less affluent mother-child dyads and households might be important in increasing optimal breastfeeding practices. Further studies might be needed to explore regional and ethnic disparities in EIB.

\section{Strengths and Weakness of the Study}

The cross-sectional nature of the study reflects only socioeconomic inequalities in EIB at a point in time. Other potential confounders such as cultural believe on the use or effect of the first breast milk (colostrum) by mothers in different communities was not adjusted for and this might have influenced the results of this study. Both the outcome and the exposure variables were self-reported, measured using a questionnaire; hence recall bias and misclassification of the exposure or the outcome with respect to the outcome and vice-versa cannot be ruled out. However this study might be among a handful of studies that provided nationally representative evidence on socio-economic inequalities in EIB among children less than 2 years of age in Uganda.

\section{Acknowledgements}

We wish to acknowledge Victoria University Kampala for providing an institutional support and amble environment for scientific research.

\section{References}

[1] Lassi, Z. S, et al, Interventions to improve neonatal health and later survival: an overview of systematic reviews. EBioMedicine, 2015. 2 (8): p. 985-1000.

[2] Fawzi, W. W, Timing of initiation, patterns of breastfeeding, and infant survival: prospective analysis of pooled data from three randomised trials. 2016.

[3] Debes, A. K, et al, Time to initiation of breastfeeding and neonatal mortality and morbidity: a systematic review. BMC public health, 2013. 13 (3): p. S19.

[4] Rollins, N. C, et al, Why invest, and what it will take to improve breastfeeding practices? The Lancet, 2016. 387 (10017): p. 491-504.

[5] Victora, C. G, et al, Breastfeeding in the 21st century: epidemiology, mechanisms, and lifelong effect. The Lancet, 2016. 387 (10017): p. 475-490.

[6] Group, N. S, Timing of initiation, patterns of breastfeeding, and infant survival: prospective analysis of pooled data from three randomised trials. The Lancet Global Health, 2016. 4
(4): p. e266-e275.

[7] Brandtzaeg, P, Mucosal immunity: integration between mother and the breast-fed infant. Vaccine, 2003. 21 (24): p. 3382-3388.

[8] Much, D, et al, Lactation is associated with altered metabolomic signatures in women with gestational diabetes. Diabetologia, 2016. 59 (10): p. 2193-2202.

[9] Hansen, K, Breastfeeding: a smart investment in people and in economies. The Lancet, 2016. 387 (10017): p. 416.

[10] Debes, A. K, et al, Time to initiation of breastfeeding and neonatal mortality and morbidity: a systematic review. 2013. 13 (3): p. S19.

[11] Bhutta, Z. A, et al, Can available interventions end preventable deaths in mothers, newborn babies, and stillbirths, and at what cost? 2014. 384 (9940): p. 347-370.

[12] Victora, C. G, et al, Breastfeeding in the 21st century: epidemiology, mechanisms, and lifelong effect. 2016. 387 (10017): p. 475-490.

[13] Rollins, N. and T. Doherty, Improving breastfeeding practices at scale. The Lancet Global Health, 2019.7 (3): p. e292-e293.

[14] Chowdhury, R, et al, Breastfeeding and maternal health outcomes: a systematic review and meta - analysis. 2015. 104: p. 96-113.

[15] Collective, G. B, UNICEF, and W. H. Organization, Global breastfeeding scorecard, 2017: Tracking progress for breastfeeding policies and programmes. World Health Organization, 2017.

[16] UNICEF, W. a, Enabling women to breastfeed through better policies and programmes: Global breastfeeding scorecard 2018. 2018.

[17] Issaka, A. I, K. E. Agho, and A. M. Renzaho, Prevalence of key breastfeeding indicators in 29 sub-Saharan African countries: a meta-analysis of demographic and health surveys (2010-2015). BMJ open, 2017.7 (10): p. e014145.

[18] ICF, U, of S. (UBOS) and. (2018). Uganda Demographic Health Survey 2016. Uganda Demographic and Health Survey 2016.

[19] Collective, G. B. and UNICEF, Nurturing the health and wealth of nations: the investment case for breastfeeding. World Health Organization, 2017.

[20] John, J. R, et al, Determinants of early initiation of breastfeeding in Ethiopia: a population-based study using the 2016 demographic and health survey data. BMC pregnancy and childbirth, 2019. 19 (1): p. 69.

[21] Ogunlesi, T. A, Maternal socio-demographic factors influencing the initiation and exclusivity of breastfeeding in a Nigerian semi-urban setting. Maternal and child health journal, 2010. 14 (3): p. 459-465.

[22] Acharya, P. and V. Khanal, The effect of mother's educational status on early initiation of breastfeeding: further analysis of three consecutive Nepal Demographic and Health Surveys. BMC Public Health, 2015. 15 (1): p. 1069.

[23] Wilkinson, R. G. and M. Marmot, Social determinants of health: the solid facts. 2003: World Health Organization. 
[24] Marmot, M, Social determinants of health inequalities. The lancet, 2005. 365 (9464): p. 1099-1104.

[25] Sharma, I. K. and A. Byrne, Early initiation of breastfeeding: a systematic literature review of factors and barriers in South Asia. International breastfeeding journal, 2016. 11 (1): p. 17.

[26] Kimani-Murage, E. W, et al, Factors affecting actualisation of the WHO breastfeeding recommendations in urban poor settings in K enya. Maternal \& Child Nutrition, 2015. 11 (3): p. $314-332$.

[27] Gertz, B. and E. DeFranco, Predictors of breastfeeding non initiation in the NICU. Maternal \& child nutrition, 2019: p. e12797.

[28] Wagner, S, et al, Breastfeeding initiation and duration in France: The importance of intergenerational and previous maternal breastfeeding experiences-results from the nationwide ELFE study. Midwifery, 2019. 69: p. 67-75.

[29] Yngve, A. and M. Sjöström, Breastfeeding determinants and a suggested framework for action in Europe. Public health nutrition, 2001. 4 (2b): p. 729-739.

[30] Bbaale, E, Determinants of early initiation, exclusiveness, and duration of breastfeeding in Uganda. Journal of health, population, and nutrition, 2014. 32 (2): p. 249.

[31] Kalisa, R, et al, Magnitude and factors associated with delayed initiation of breastfeeding among mothers who deliver in Mulago hospital, Uganda. African health sciences, 2015. 15 (4): p. 1130-1135.

[32] Statistics, U. B. o. and ICF, Uganda Demographic and Health Survey 2016: Key Indicators Report. 2017, Uganda Bureau of Statistics (UBOS), and Rockville, MD: UBOS and ICF Kampala.

[33] Organization, W. H, Indicators for assessing infant and young child feeding practices: part 2: measurement. 2010.
[34] Uganda, M, Policy Guidelines on Infant and Young Child Feeding. January 2009. 2009.

[35] Elias, P, Occupational Classification (ISCO-88). 1997.

[36] Vyas, S, L. J. H. p. Kumaranayake, and planning, Constructing socio-economic status indices: how to use principal components analysis. 2006. 21 (6): p. 459-468.

[37] ICF, U. J. U. D. and H. Survey, of S. (UBOS) and. (2018). Uganda Demographic Health Survey 2016.

[38] Organization, W. H, Indicators for assessing infant and young child feeding practices: part 1: definitions: conclusions of a consensus meeting held 6-8 November 2007 in Washington DC, USA. 2008.

[39] Organization, W. H, Indicators for assessing infant and young child feeding practices: conclusions of a consensus meeting held 6-8 November 2007 in Washington DC, USA. 2008: World Health Organization (WHO).

[40] Ndirangu, M, et al, Trends and factors associated with early initiation of breastfeeding in Namibia: analysis of the Demographic and Health Surveys 2000-2013. 2018. 18 (1): p. 171.

[41] Ndirangu, M, et al, Trends and factors associated with early initiation of breastfeeding in Namibia: analysis of the Demographic and Health Surveys 2000-2013. BMC pregnancy and childbirth, 2018. 18 (1): p. 171.

[42] Berde, A. S. and S. S. Yalcin, Determinants of early initiation of breastfeeding in Nigeria: a population-based study using the 2013 demograhic and health survey data. BMC Pregnancy and Childbirth, 2016. 16 (1): p. 32.

[43] Houweling, T. A, et al, Huge poor-rich inequalities in maternity care: an international comparative study of maternity and child care in developing countries. 2007. 85: p. 745-754. 